\title{
CC and NC Pion Production
}

\author{
Alejandro Mariano ${ }^{1,2^{*}}$, Cesar Barbero ${ }^{1,2}$, Gabriel López-Castro ${ }^{3}$ \\ ${ }^{1}$ Departamento de F sica, Facultad de Ciencias Exactas, Universidad Nacional de La Plata, Buenos Aires, Argentina \\ ${ }^{2}$ Instituto de Fsica La Plata, CONICET, Buenos Aires, Argentina \\ ${ }^{3}$ Departamento de F sica, Cinvestav, Mexico City, Mexico \\ Email: ${ }^{*}$ mariano@fisica.unlp.edu.ar
}

Received October 2, 2013; revised November 1, 2013; accepted November 28, 2013

Copyright (C) 2013 A. Mariano et al. This is an open access article distributed under the Creative Commons Attribution License, which permits unrestricted use, distribution, and reproduction in any medium, provided the original work is properly cited. In accordance of the Creative Commons Attribution License all Copyrights (C) 2013 are reserved for SCIRP and the owner of the intellectual property A. Mariano et al. All Copyright (C) 2013 are guarded by law and by SCIRP as a guardian.

\begin{abstract}
The disappearance searching experiments $v_{\mu} \rightarrow v_{x}$ use charged current quasielastic (CCQE) reaction to detect an arriving neutrino and reconstruct its energy, while the $\mathrm{CC} 1 \pi^{+}$production can mimic the $\mathrm{CCQE}$ signal process. In $v_{\mu} \rightarrow v_{e}$ appearance experiments, the $\mathrm{NC} 1 \pi^{0}$ production process can lead to a fake $e^{-}$event by the impossibility for the detector of distinguish an arriving electron or a photon. Here we present a consistent model, from the point of view of the construction of the elemental amplitude, for the mentioned pion production background processes including bounding, smearing and final state interaction (FSI) effects in a single fashion. Results are comparable with more evolved approaches based on Monte Carlo simulations.
\end{abstract}

Keywords: Neutrino Scattering; Pion Production; Neutrino Oscillation

\section{Introduction}

Neutrino oscillation experiments search a distortion in the neutrino flux at a detector positioned far away (L) from the source. By comparing near and far neutrino energy spectra, one gains information about the oscillation probability

$$
P\left(v_{i} \rightarrow v_{j}\right)=\frac{\sin ^{2} 2 \theta_{i j} \sin ^{2} \Delta m_{i, j}^{2} L}{2 E_{v}},
$$

and then about the $\theta_{i j}$ mixing angles and $\Delta m_{i, j}^{2}$ mass squared differences. New high quality data are becoming from MiniBoone, MINOS, NOMAD, MinervA and SciBoone full dedicated to measure cross sections.

Disappearance searching experiments $v_{\mu} \rightarrow v_{x}$ use $v_{\mu} n \rightarrow \mu^{-} p$ CCQE reaction to detect an arriving neutrino and reconstruct its energy. $E_{v}$ determination could be wrong for a fraction of $\mathrm{CC} 1 \pi^{+}$background events $(20 \%) \quad v_{\mu} p \rightarrow \mu^{-} p \pi^{+}$, that can mimic a CCQE one if the pion is absorbed in the target and/or not detected. In $v_{\mu} \rightarrow v_{e}$ appearance experiment, one detects $v_{e}$ in an (almost) $v_{\mu}$ beam. Here the signal event $v_{e} n \rightarrow e^{-} p$ is dominated by a $\mathrm{NC} 1 \pi^{0} \quad v_{\mu} N \rightarrow v_{\mu} N \pi^{0}$

${ }^{*}$ Corresponding author. background, and the detector can not distinguish between $e^{-}$and $\pi^{0}$ if one of both photons from the $\pi^{0} \rightarrow \gamma \gamma$ decay escapes. Then a precise knowledge of cross sections is a prerequisite in order to make simulations in event generators to substract fake $1 \pi$ events in QE countings.

Several models have been developed over the last thirty years to evaluate these corresponding background elementary cross sections [1-4]. The scattering amplitude in all these models always contains a resonant term (R) in the $\pi N$ system, described by the $\Delta(1232)$-pole contribution and (in some cases) by higher mass intermediate resonances, plus a nonresonant (B) term describing other processes (where the cross- $\Delta$ contribution can also be included) leading to $\pi N$ final states. The differences between all these models stem mainly from the treatment of the vertexes and the propagator used to describe the $\Delta$ resonance and from the consideration (or not) of the nonresonant terms and its interference with the resonant contribution. Nuclear effects and FSI have been introduced by several works, where different nuclear models and event generators or simulations codes have been implemented in [5] (GiBUU) and $[6,7]$. 
In this paper we reanalyze the elementary amplitude, bounding + ground state correlations (GSC) effects, and FSI on the emerging nucleon $(\mathrm{N})$ and pion $(\pi)$, all what will be developed in the following sections.

\section{Elementary Amplitude}

For the $\nu N \rightarrow l N^{\prime} \pi$ process the total cross section reads

$$
\begin{aligned}
\sigma\left(E v^{C M}\right)= & \frac{F^{C C / N C}}{(2 \pi)^{4} E_{v}^{C M} \sqrt{s}} \int_{E_{l}-}^{E_{l}^{+}} \mathrm{d} E_{l}^{C M} \int_{E_{\pi}^{-}}^{E_{\pi}^{+}} \mathrm{d} E_{\pi}^{C M} \\
& \cdot \int_{-1}^{+1} \mathrm{~d} \cos \theta \int_{0}^{2 \pi} \mathrm{d} \eta \frac{1}{16} \sum_{\text {spin }}|M|^{2},
\end{aligned}
$$

being $E_{v}^{C M}=\frac{m_{N} E_{v}^{L a b}}{\sqrt{2 E_{v}^{L a b} m_{N}+m_{N}^{2}}}$ is the CM-Lab $v$-en-

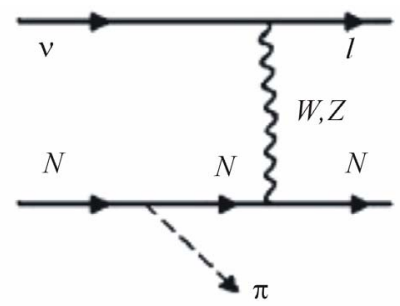

(a)

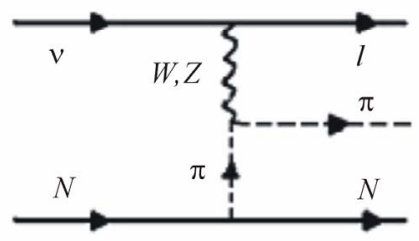

(c)

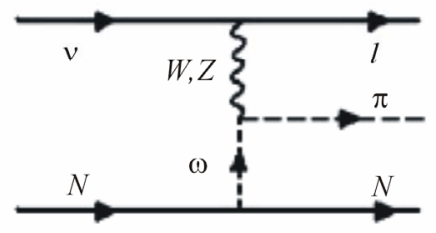

(e)

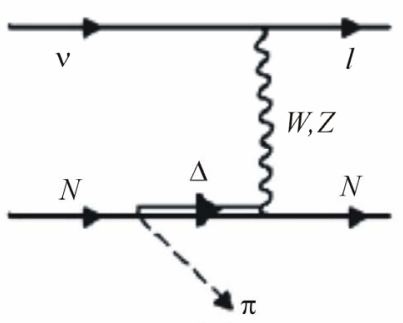

(g) ergy relation, $l$ the merging lepton, $F^{C C / N C}$ phasespace factors, and

$$
\mathcal{M}=\mathcal{M}_{B}+\mathcal{M}_{R}, \quad R \equiv \Delta .
$$

the amplitude where the contributions for the $B$ amplitude are shown in the Figures 1(a)-(g), while for the $R$ contribution is shown in the Figure 1(h).

The requirements on the hadronic part of the amplitude

$$
\begin{aligned}
\mathcal{M}_{i}= & -\frac{G_{F}}{\sqrt{2}} \bar{u}\left(p_{l}^{\prime}\right)(-i) \gamma_{\lambda}\left(1-\gamma_{5}\right) u\left(p_{v}\right) \\
& \times \bar{u}\left(p^{\prime}\right)\left(\mathcal{O}_{V i}^{\lambda}-\mathcal{O}_{A i}^{\lambda}\right) u(p), i=B, R
\end{aligned}
$$

where $u$ indicate the lepton and nucleon spinors, are: 1) Unitarity, violated with real $B$ terms. It is possible an unitarization by introduction of experimental phase shifts

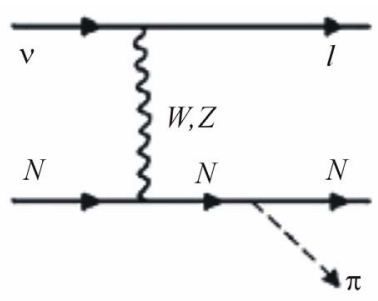

(b)

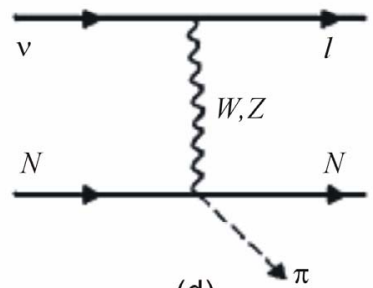

(d)

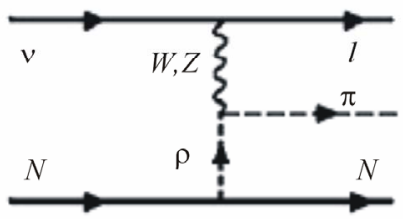

(f)

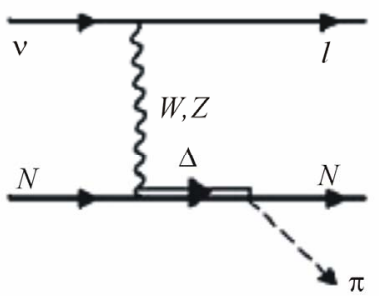

(h)

Figure 1. Different contributions to the amplitude. 
and rescattering of the final $\pi N$ pair, but the effects are not so important as in photoproduction.

2) Vector amplitude should fulfill electromagnetic gauge invariance (GI) $\rightarrow \bar{u} \mathcal{O}_{3 i}^{\lambda} q_{\lambda} u=0$. In the $B$ amplitude we must to have same vector FF ( $\rho$ contribution is axial and the $\omega$ one is self-GI), while for $R$ contributions GI must be fulfilled in presence of finite width effects.

3) $\mathcal{M}_{R}(S=3 / 2)$ should be invariant under contact transformations (CT), which fixes the Feynman rules to built the amplitude [8], being the corresponding propagator (in the momentum space)

$$
\begin{aligned}
G\left(p,-\frac{1}{3}\right)_{\mu \nu}= & -\left[\frac{p+m}{p^{2}-m^{2}} \hat{P}_{\mu \nu}^{3 / 2}+\frac{2}{m^{2}}(\not p+m)\right. \\
& \left.\times\left(\hat{P}_{11}^{1 / 2}\right)_{\mu \nu}+\frac{\sqrt{3}}{m}\left(\hat{P}_{12}^{1 / 2}+\hat{P}_{21}^{1 / 2}\right)_{\mu \nu}\right],
\end{aligned}
$$

and a general vertex interaction

$$
\Gamma=g \bar{\psi}_{\mu} F^{\mu}(\psi, \Psi, \phi, \cdots)+\text { h.c. },
$$

depending on the fields (nucleon, pion, photon, $\mathrm{W}-\mathrm{Z}$ bosons, etc.) interacting with the $\Delta$ field $\bar{\psi}_{\mu}$. We have introduced $P_{i j}^{k}$ (defined in Ref. [9]) which projects on the $k=3 / 2,1 / 2$ space. Unstableness of $\Delta$ is included in the $G_{\mu v}(p)$ through a self-energy $\Sigma_{\mu v}(p)$ (one loop-corrections), which accounts an energy dependent width and vertex corrections to get GI. Alternatively, we make $G^{\text {dressed }} \approx G\left(m_{\Delta} \rightarrow m_{\Delta}-i \Gamma_{\Delta} / 2\right)$, referred as complex mass scheme that results to be appropriated along the resonance region [10].

\section{Bounding + GSC + FSI}

The bounding effects in the nucleus are introduced within the relativistic Hartree approximation (RHA) of QHD I where the exchange of $\sigma, \omega$ mesons is considered. The meson fields are approximated by their vacuum spectation (MFT), i.e. constant values, and within the RHA [11] the vacuum fluctuation corrections are added. The nucleon field is expanded as

$$
\begin{aligned}
\psi_{N}(x)= & \int \mathrm{d} p^{3} \sum_{m_{s} m_{t}} \sqrt{m_{N}^{*}(2 \pi)^{3} E^{*}(\mathbf{p})}\left[u\left(\mathbf{p} m_{s} m_{t}\right) a_{\mathbf{p} m_{s} m_{t}}\right. \\
& \left.\times \mathrm{e}^{i p \cdot x}+b_{\mathbf{p} m_{s} m_{t}}^{\dagger} v\left(\mathbf{p} m_{s} m_{t}\right) \mathrm{e}^{-i p \cdot x}\right],
\end{aligned}
$$

being the single particle spectrum

$$
p_{0}=C_{V}^{2} \rho_{B} m_{N}^{2}+E^{*}(\mathbf{p}) \equiv \Sigma_{0}^{V}\left(C_{V}\right)+E^{*}(\mathbf{p}),
$$

where

$$
E^{*}(\mathbf{p})=\sqrt{\mathbf{p}^{2}+m_{N}^{* 2}}, m_{N}^{*} \equiv m_{N}+\Sigma^{S}\left(C_{S}, m_{N}^{*}\right) .
$$

$m_{N}^{*}<m_{N}$ is the effective mass acquired by the nucleon [11] through the scalar self-energy $\Sigma \equiv \Sigma^{S} . \Sigma_{M F T}^{S}$ in- cludes the tadpole diagram from Figure 2, retaining in its evaluation only the contribution from nucleons in the filled Fermi sea in the nucleon propagator (tick full lines). $\Sigma_{R H A}^{S}$ includes the same diagram but the full nucleon propagator (which encloses the contribution of the occupied negative-energy states) is used in the evaluation of the self-energy. $C_{V}$ and $C_{S}$ are fixed to reproduce the experimental binding energy per nucleon of $-16 \mathrm{MeV}$ at the Fermi momentum $p_{F}=1.42 \mathrm{fm}^{-1}$ for the normal nuclear matter. For the $\Delta$ we assume the same scalar and vector self energies that for nucleon, approximation known as "universal couplings". In the structure of the ground state, $2 \mathrm{p} 2 \mathrm{~h}+4 \mathrm{p} 4 \mathrm{~h}$ states $(p, h \equiv$ particle, hole) are included through perturbation theory in nuclear matter, from which a momentum distribution can be built as

$$
\begin{gathered}
n_{A}(\mathbf{k})=\left\langle\tilde{0}\left|a_{\mathbf{k} m}^{\dagger} a_{\mathbf{k} m}\right| \tilde{0}\right\rangle, \int \mathrm{d}^{3} k n_{A}(\mathbf{k})=\frac{A}{4} \\
|\tilde{0}\rangle=\mathcal{N}\left[|0\rangle+\frac{1}{(2 !)^{2}} \sum_{p^{\prime} s, h^{\prime} s} c_{p_{1} p_{2} h_{1} h_{2}}\left|p_{1} p_{2} h_{1} h_{2}\right\rangle\right. \\
\left.+\frac{1}{(4 !)^{2}} \sum_{p^{\prime} s, h^{\prime} s} c_{p_{1} p_{2} p_{3} p_{4} h_{1} h_{2} h_{3} h_{4}}\left|p_{1} p_{2} p_{3} p_{4} h_{1} h_{2} h_{3} h_{4}\right\rangle\right],
\end{gathered}
$$

where

$$
\begin{gathered}
c_{p_{1} p_{2} h_{1} h_{2}}=-\frac{\left\langle p_{1} p_{2} h_{1} h_{2}|\hat{V}| 0\right\rangle}{E_{p_{1} p_{2} h_{1} h_{2}}}, \\
c_{p_{1} p_{2} p_{3} p_{4} h_{1} h_{2} h_{3} h_{4}}= \\
\frac{\left\langle 0|\hat{V}| p_{1} p_{2} h_{1} h_{2}\right\rangle\left\langle p_{1} p_{2} h_{1} h_{2}|\hat{V}| p_{1} p_{2} p_{3} p_{4} h_{1} h_{2} h_{3} h_{4}\right\rangle}{E_{p_{1} p_{2} h_{1} h_{2}} E_{p_{1} p_{2} p_{3} p_{4} h_{1} h_{2} h_{3} h_{4}}} .
\end{gathered}
$$

FSI on nucleons are taken (Toy model!) through the used effective fields within the RHA also for final $\mathrm{N}$. While for pions we use the Eikonal approach in its simplest version [12], that is $\phi_{\pi} \rightarrow \phi_{\pi}^{*}$, where

$$
\phi_{\pi}^{*}(\mathbf{r}) \sim \mathrm{e}^{-i \mathbf{p} \mathbf{p}_{\pi} \cdot \mathbf{r}} \mathrm{e}^{-i / v_{\pi} \int_{z_{\pi}}^{\infty} V_{o p t}\left(\mathbf{b}, \mathbf{z}^{\prime}\right) \mathrm{d} \mathbf{z}^{\prime}}, \mathbf{r}=\left(\mathbf{b}, \mathbf{z}^{\prime}\right) .
$$

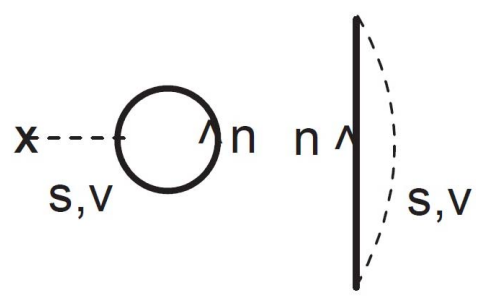

(a)

(b)

Figure 2. (a) Tadpole diagram included in the MFT and RHA self-energies. (b) Tadpole exchange diagram that is added in order to get the relativistic Hartree Fock self-energy. The dashed lines indicate the propagator of the scalar (S) or vector meson (V) that interacts with a nucleon $n$ (full lines). 
Assuming a mean distance of trip for $\pi$ in nucleus $(\langle d\rangle)$, constant nucleon density and the $\Delta$-h model for the $\pi$-optical potential, we get

$$
\begin{aligned}
& \phi_{\pi}^{*}(\mathbf{r}) \sim \mathrm{e}^{-i \mathbf{p}_{\pi} \cdot \mathbf{r}} \mathrm{e}^{-i \lambda(s)\left|\mathbf{p}_{\pi}\right|\langle d\rangle}, \\
& \lambda(s)=\frac{2}{9}\left(f_{\pi N \Delta} m_{\pi}\right)^{2} m_{N}^{2} \rho_{0} T s\left(\sqrt{s}-m_{\Delta}^{*}+1 / 2 \Gamma_{\Delta}^{*}\right), \\
& \langle d\rangle=\sqrt{R^{2}-2 / 3\langle r\rangle^{2}}, R=r_{0} A^{1 / 3},\langle r\rangle=c A^{1 / 3},
\end{aligned}
$$

where $A, R$ and $T=N, Z / A$ are the mass number, radii and isospin factor of the nucleus respectively.

\section{Results and Conclusions}

The different coupling constants presented in the B and $\mathrm{R}$ terms have been fixed by the fitting to the elastic $\pi^{+} p \rightarrow \pi^{+} p$ cross section data [13], to the $\gamma p \rightarrow \pi^{0} p$ and $\gamma p \rightarrow \pi^{+} n$ processes [14], and using the CVC hypotesis and a fitting to the differential cross section $\frac{\mathrm{d}\langle\sigma\rangle}{\mathrm{d} Q^{2}}$ for the $v p \rightarrow \mu^{-} p \pi^{+}$(ANL) data [15]. The results obtained for the background processes mentioned at the introduction are shown in Figures 3-5, where the different effects, bounding, smearing (GSC) and FSI are added gradually. In Figure 3 we show the total cross section for the $v_{\mu} A \rightarrow \mu^{-}(A-1) p \pi^{+}$process where $A={ }^{12} C+2 H$ (mineral oil detector) and compare with the corresponding data of the MoniBooNE [16,17] experiment. Results corresponding to the $v_{\mu} A \rightarrow \mu^{-}(A-1) N \pi^{0}$ reaction are shown in Figure 4, and those for NC1 $\pi^{0}$ per nucleon in Figure 5.

Calculations are $\sim 50 \%$ below MoniBooNE for $\mathrm{CC}$ $1 \pi$ (comparable to the GiBUU Monte Carlo results) and $\sim 30 \%$ for $\mathrm{NC} \pi^{0}$ production. The FSI inclusion is very primitive, and perhaps an overvaluation of them is

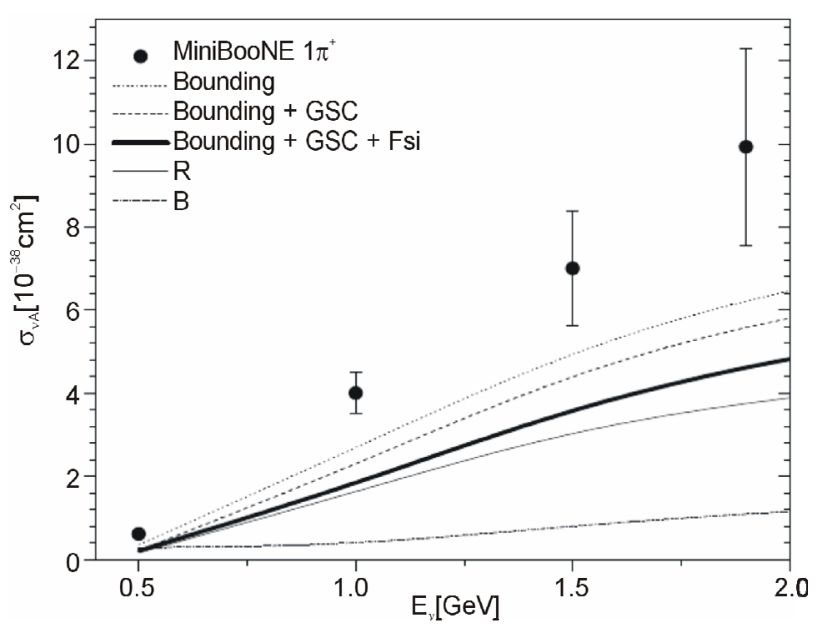

Figure 3. CC $1 \pi^{+}$calculation compared with MiniBooNE data [16].

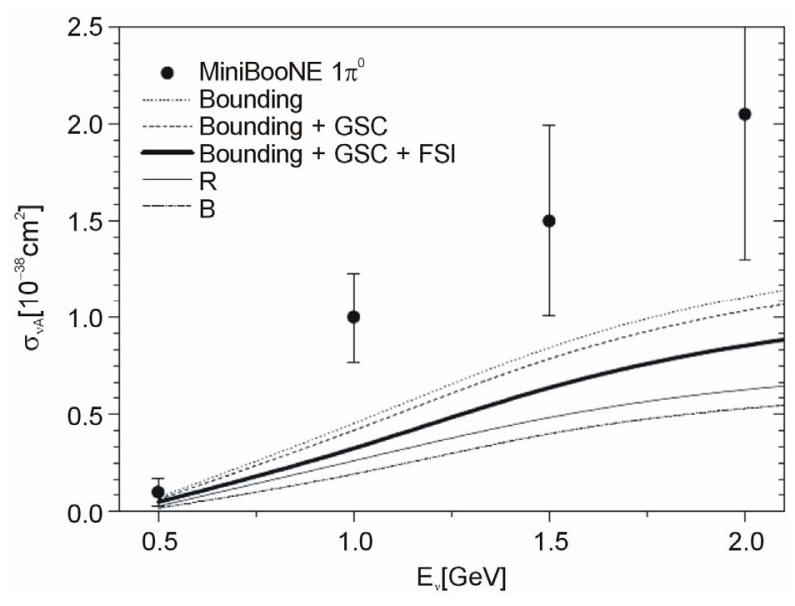

Figure 4. Idem for $\mathrm{CC} 1 \pi^{0}$.

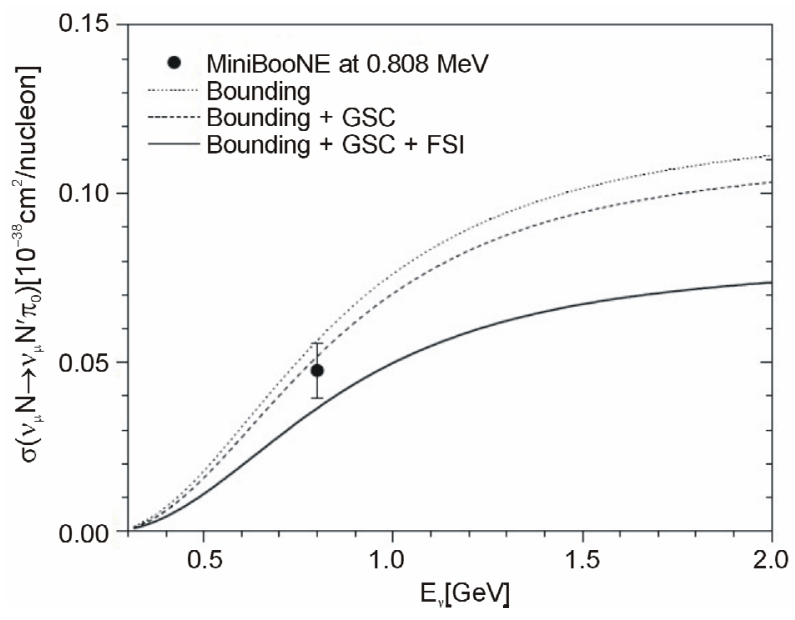

Figure 5. Idem for NC $1 \pi^{0}$. Data form [17].

presented and should be improved. Nevertheless, it is noted that for example at $E_{v}=1.5 \mathrm{GeV}$ for MiniBooNE and ANL or BNL (without cuts), data

$$
\begin{aligned}
& \sigma_{A C C 1 \pi^{+}}^{\exp } / A \sigma_{N C C 1 \pi^{+}}^{\exp } \sim 95 \%, \\
& \sigma_{A C C 1 \pi^{0}}^{\exp } / A \sigma_{N C C 1 \pi^{0}}^{\exp } \sim 83 \%, \sigma_{A N C 1 \pi^{0}}^{\exp } / A \sigma_{N N C 1 \pi^{0}}^{\exp } \sim 92 \% .
\end{aligned}
$$

This seems to indicate that nuclear effects should be of much minor importance, or that another mechanism coming from nuclear effects should be considered, as $2 \mathrm{p} 2 \mathrm{~h}+1 \pi$ configurations generated by FSI added to the $1 \mathrm{p} 1 \mathrm{~h}+1 \pi$ considered here, and meson exchange currents contributions that are also capable of generating $2 \mathrm{p} 2 \mathrm{~h}+$ $1 \pi$ acting on the nuclear ground state.

\section{REFERENCES}

[1] G. L. Fogli and G. Nardulli, Nuclear Physics B, Vol. 160, 1979, pp. 116-150. http://dx.doi.org/10.1016/0550-3213(79)90233-5

[2] T. Sato, D. Uno and T.-S. H. Lee, Physical Review C, Vol. 67, 2003, Article ID: 065201. 
http://dx.doi.org/10.1103/PhysRevC.67.065201

[3] E. Hernandez, J. Nieves and M. Valverde, Physical Review D, Vol. 76, 2007, Article ID: 033005.

http://dx.doi.org/10.1103/PhysRevD.76.033005

[4] O. Lalakulich, T. Leitner, O. Buss and U. Mosel, Physical Review D, Vol. 82, 2010, Article ID: 093001. http://dx.doi.org/10.1103/PhysRevD.82.093001

[5] O. Lalakulich and U. Mosel, Pion Production in the MiniBooNE Experiment, Physical Review C, Vol. 87, 2013, Article ID: 014602.

http://dx.doi.org/10.1103/PhysRevC.87.014602

[6] J. T. Sobczyk and J. Zmuda, Physical Review C, Vol. 87, 2013, Article ID: 065503. http://dx.doi.org/10.1103/PhysRevC.87.065503

[7] E. Hernandez, J. Nieves and M. J. Vacas, Physical Review D, 87, 2013, Article ID: 113009. http://dx.doi.org/10.1103/PhysRevD.87.113009

[8] M. El-Amiri, G. López Castro and J. Pestieau, Nuclear Physics A, Vol. 543, 1992, pp. 673-684. http://dx.doi.org/10.1016/0375-9474(92)90553-V

[9] F. de Jong and R. Malfliet, Physical Review C, Vol. 46, 1992, pp. 2567-2581. http://dx.doi.org/10.1103/PhysRevC.46.2567
[10] C. Barbero, A. Mariano and G. López Castro, Journal of Physics G: Nuclear and Particle Physics, Vol. 39, 2012, Article ID: 085011. http://dx.doi.org/10.1088/0954-3899/39/8/085011

[11] J. D. Walecka, "Theroretical Nuclear and Subnuclear Physics," Part I, Oxford University Press, New York, 1995.

[12] C. Barbero, A. Mariano and S. B. Duarte, Physical Review $C$, Vol. 82, 2010, Article ID: 067305. http://dx.doi.org/10.1103/PhysRevC.82.067305

[13] G. López Castro and A. Mariano, Nuclear Physics A, Vol. 697, 2001, pp. 440-468.

[14] A. Mariano, Journal of Physics G: Nuclear and Particle Physics, Vol. 34, 2007, p. 1627.

[15] C. Barbero, G. López Castro and A. Mariano, Physics Letters B, Vol. 664, 2008, pp. 70-77. http://dx.doi.org/10.1016/j.physletb.2008.05.011

[16] A. A. Aguilar-Arevalo, et al., Physical Review D, Vol. 83, 2011, Article ID: 052007. http://dx.doi.org/10.1103/PhysRevD.83.052007

[17] A. A. Aguilar-Arevalo, et al., Physical Review D, Vol. 81, 2010, Article ID: 013005. http://dx.doi.org/10.1103/PhysRevD.81.013005 